\title{
TIME-FREQUENCY-MOVING-AVERAGE PROCESSES: PRINCIPLES AND CEPSTRAL METHODS FOR PARAMETER ESTIMATION
}

\author{
Michael Jachan, Gerald Matz, and Franz Hlawatsch \\ Institute of Communications and Radio-Frequency Engineering, Vienna University of Technology \\ Gusshausstrasse 25/389, A-1040 Wien, Austria (Europe) \\ phone: +43 158801 38914, fax: +43 158801 38999, email: michael.jachan@tuwien.ac.at \\ web: www.nt.tuwien.ac.at/dspgroup/time.html
}

\begin{abstract}
We introduce the time-frequency-moving-average (TFMA) model as a highly parsimonious time-varying MA model formulated in terms of time-frequency (TF) shifts. For estimation of the TFMA model parameters, we develop a computationally efficient nonlinear technique based on a novel complex TF cepstrum, TF cepstral recursions, and an underspread approximation. Simulation results demonstrate significant performance advantages of the proposed TFMA model and parameter estimation technique over an existing method for time-varying MA modeling and estimation.
\end{abstract}

\section{INTRODUCTION}

Autoregressive (AR) and moving average (MA) models for random processes are useful in many signal processing applications. Timevarying models are applicable to a wider class of problems than are their time-invariant counterparts. In particular, time-varying MA models are formulated as (see e.g. [1] and references therein)

$$
x[n]=\sum_{m=0}^{M} b_{m}[n] e[n-m]=\sum_{m=0}^{M} b_{m}[n]\left(\mathbb{S}^{m} e\right)[n],
$$

where the $b_{m}[n], m=0, \ldots, M$ (with $b_{0}[n]>0$ ) are the time-varying MA parameters, $M$ is the MA model order (maximum delay), $e[n]$ is stationary white noise with unit variance, and $\mathbb{S}$ denotes the timeshift operator defined as $(\mathbb{S} x)[n]=x[n-1]$. We can interpret (1) as the input-output relation $x[n]=\sum_{m=0}^{M} H[n, m] e[n-m]$ of a linear time-varying (LTV) system-known as the innovations systemwith impulse response $H[n, m]=b_{m}[n]$. A cepstral technique for estimating the parameters $b_{m}[n]$ has recently been developed [2].

Here, we propose the highly parsimonious time-frequency $(T F)$ MA model that complements the TFAR model presented in [3]. The TFMA model is introduced in Section 2. In Section 3, we define the complex TF cepstrum and derive a TF cepstral recursion which is used in Section 4 to develop a computationally efficient parameter estimation technique. Simulation results presented in Section 5 demonstrate significant performance advantages of our estimation technique over the technique proposed in [2].

\section{THE TFMA MODEL}

In the following, the signal $x[n]$ will be considered on the interval $[0, N-1]$ with $N$ even. Motivated by the observation that LTV systems cause frequency shifts of the input signal in addition to time shifts, we define the TFMA model of $\operatorname{order}(M, L)$ as (see Fig. 1)

$$
x[n]=\sum_{m=0}^{M} \sum_{l=-L}^{L} b_{m, l}\left(\mathbb{M}^{l} \mathbb{S}^{m} e\right)[n]=\sum_{m=0}^{M} \sum_{l=-L}^{L} b_{m, l} e^{j \frac{2 \pi}{N} l n} e[n-m],
$$

where $\mathbb{M}$ denotes the frequency-shift operator defined as $(\mathbb{M} x)[n]=$ $e^{j \frac{2 \pi}{N} n} x[n]$ and $L$ is the spectral (Doppler) model order. Note that the parameters $b_{m, l}$ do not depend on time $n$, and that the time-invariant

Funding by FWF grant P15156.

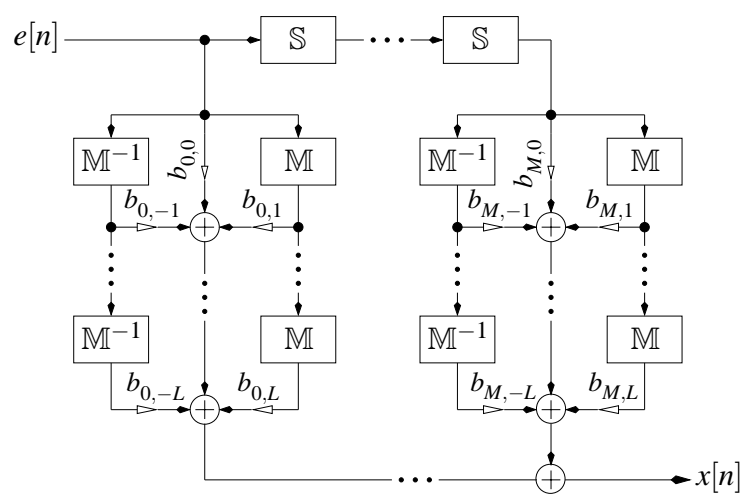

Figure 1: Block diagram of the TFMA model of order $(M, L)$.

MA model [4] is reobtained for $L=0$. Furthermore, the TFMA model and the time-varying MA model (1) are related via the basis expansion [1]

$$
b_{m}[n]=\sum_{l=-L}^{L} b_{m, l} e^{j \frac{2 \pi}{N} l n} .
$$

We assume that $b_{0, l}$ is an autocorrelation sequence such that $b_{0}[n]>$ 0 . We also suppose that the TFMA process $x[n]$ is underspread [5] in the sense that $4(2 L+1)(M+1) \leq N$, i.e., the number of TFMA parameters $b_{m, l}$ in (2) is at most $N / 4$.

The input-output relation of the LTV innovations system underlying the TFMA model can be written as a superposition of TFshifted versions of the input signal [6]:

$$
x[n]=\frac{1}{N} \sum_{m=-N / 2}^{N / 2-1} \sum_{l=-N / 2}^{N / 2-1} S[m, l] e^{j \frac{2 \pi}{N} l n} e[n-m] .
$$

Here, $S[m, l]$ is the system's spreading function (SF) defined as ${ }^{1}$

$$
S[m, l] \triangleq \sum_{n=0}^{N-1} H[n, m] e^{-j \frac{2 \pi}{N} l n}=\underset{n \rightarrow l}{\mathbb{F}} H[n, m] .
$$

Comparing (4) with (2), we see that the SF of the innovations system essentially equals the TFMA parameters $b_{m, l}$ :

$$
S[m, l]= \begin{cases}N b_{m, l}, & (m, l) \in[0, M] \times[-L, L] \\ 0, & \text { otherwise }\end{cases}
$$

A TFMA model will be called minimum phase if the underlying innovations system is minimum phase, i.e., if $H[n, m]$ is minimum phase in $m$ for all $n$, or equivalently (cf. (5)) if $S[m, l]$ is minimum phase in $m$ for all $l$.

${ }^{1}$ When using the discrete Fourier transform (DFT) $\mathbb{F}$ or the inverse DFT $\mathbb{F}^{-1}$, the function being transformed is assumed periodic with period $N$. 


\section{TF CEPSTRUM AND TF CEPSTRAL RECURSION}

As a basis for the parameter estimation technique proposed in Section 4, we now introduce the complex TF cepstrum (CTFC) and derive a $T F$ cepstral recursion. This generalizes classical cepstrum theory $[7,8]$ to the time-varying case. We also discuss the relation of the CTFC with the evolutionary cepstrum introduced in [2].

\subsection{The Complex TF Cepstrum}

We consider a possibly noncausal LTV system (i.e., $H[n, m]$ and $S[m, l]$ may be nonzero for $m<0)$. A time-varying transfer function can be defined as the two-sided 2-D $z$-transform [9] of the SF:

$$
L\left(z_{1}, z_{2}\right) \triangleq \frac{1}{N} \sum_{m=-N / 2}^{N / 2-1} \sum_{l=-N / 2}^{N / 2-1} S[m, l] z_{1}^{l} z_{2}^{-m} .
$$

Note that $L\left(z_{1}, z_{2}\right)$ is analytic for all $z_{1}, z_{2}$. We assume that $L\left(z_{1}, z_{2}\right)$ $\neq 0$ in a region $\mathscr{R}=\left\{\left(z_{1}, z_{2}\right) \mid\left(1-\varepsilon_{1} \leq\left|z_{1}\right| \leq 1+\varepsilon_{1}\right) \wedge\left(1-\varepsilon_{2} \leq\right.\right.$ $\left.\left.\left|z_{2}\right| \leq 1+\varepsilon_{2}\right)\right\}$ with some positive constants $\varepsilon_{1}, \varepsilon_{2}$; this guarantees that $\log \left(L\left(z_{1}, z_{2}\right)\right)$ is analytic in $\mathscr{R}$. We then define the CTFC $s[m, l]$ implicitly as (cf. [10])

$$
\log \left(L\left(z_{1}, z_{2}\right)\right)=\frac{1}{N} \sum_{m=-\infty}^{\infty} \sum_{l=-\infty}^{\infty} s[m, l] z_{1}^{l} z_{2}^{-m} .
$$

The CTFC is a function of delay $m$ and Doppler shift $l$; it is causal (i.e., $s[m, l]=0$ for $m<0$ ) if and only if the SF $S[m, l]$ is minimum phase (and, thus, also causal) with respect to $m$ for all $l$ (cf. [7]). If the system is time-invariant, then $S[m, l]=0$ for $l \neq 0$ and thus the CTFC reduces to the classical complex cepstrum $[7,8]$.

To compute the CTFC using DFTs, we consider

$$
L[n, k] \triangleq L\left(e^{j \frac{2 \pi}{N} n}, e^{j \frac{2 \pi}{N} k}\right)=\frac{1}{N} \sum_{m=-N / 2}^{N / 2-1} \sum_{l=-N / 2}^{N / 2-1} S[m, l] e^{-j \frac{2 \pi}{N}(k m-n l)}
$$

for $(n, k) \in[0, N-1] \times[0, N-1]$. Here, $n$ and $k$ are discrete time and discrete frequency, respectively. Equation (8) yields

$$
\begin{aligned}
\log (L[n, k]) & =\frac{1}{N} \sum_{m=-\infty}^{\infty} \sum_{l=-\infty}^{\infty} s[m, l] e^{-j \frac{2 \pi}{N}(k m-n l)} \\
& =\frac{1}{N} \sum_{m=-N / 2}^{N / 2-1} \sum_{l=-N / 2}^{N / 2-1} \tilde{s}[m, l] e^{-j \frac{2 \pi}{N}(k m-n l)},
\end{aligned}
$$

where $\tilde{s}[m, l] \triangleq \sum_{i=-\infty}^{\infty} \sum_{j=-\infty}^{\infty} s[m+i N, l+j N]$ is an aliased version of the CTFC $s[m, l]$. This aliased CTFC can hence be calculated as

$$
\begin{aligned}
\tilde{s}[m, l] & =\frac{1}{N} \sum_{n=0}^{N-1} \sum_{k=0}^{N-1} \log (L[n, k]) e^{j \frac{2 \pi}{N}(m k-l n)} \\
& =\underset{n \rightarrow l}{\mathbb{F}} \underset{k \rightarrow m}{\mathbb{F}} \mathbb{F}^{-1} \log \left(\underset{m^{\prime} \rightarrow k}{\mathbb{F}} \underset{l^{\prime} \rightarrow n}{\mathbb{F}^{-1}} S\left[m^{\prime}, l^{\prime}\right]\right),
\end{aligned}
$$

where (9) has been used. Aliasing is negligible (i.e., $\tilde{s}[m, l] \approx s[m, l]$ for $(m, l) \in[-N / 2, N / 2-1] \times[-N / 2, N / 2-1])$ if $s[m, l]$ is sufficiently decayed for $(m, l) \notin[-N / 2, N / 2-1] \times[-N / 2, N / 2-1]$.

\subsection{The TF Cepstral Recursion}

We now assume a TFMA model that is minimum phase, so that $s[m, l]=0$ for $m<0$. Inserting (7) into (8) and using (6), we obtain

$$
\log \left(\sum_{m=0}^{M} \sum_{l=-L}^{L} b_{m, l} z_{1}^{l} z_{2}^{-m}\right)=\frac{1}{N} \sum_{m^{\prime}=0}^{\infty} \sum_{l^{\prime}=-\infty}^{\infty} s\left[m^{\prime}, l^{\prime}\right] z_{1}^{l^{\prime}} z_{2}^{-m^{\prime}}
$$

Differentiation with respect to $z_{2}^{-1}$ gives

$$
\begin{array}{r}
\sum_{m=1}^{M} \sum_{l=-L}^{L} m b_{m, l} z_{1}^{l} z_{2}^{-m+1}=\frac{1}{N} \sum_{m^{\prime}=1}^{\infty} \sum_{l^{\prime}=-\infty}^{\infty} \sum_{m=0}^{M} \sum_{l=-L}^{L} m^{\prime} b_{m, l} s\left[m^{\prime}, l^{\prime}\right] \\
\cdot z_{1}^{l+l^{\prime}} z_{2}^{-m-m^{\prime}+1} .
\end{array}
$$

By matching the powers of $z_{1}$ and $z_{2}$, we obtain the relation

$$
m b_{m, l}=\frac{1}{N} \sum_{m^{\prime}=0}^{M} \sum_{l^{\prime}=-L}^{L}\left(m-m^{\prime}\right) b_{m^{\prime}, l^{\prime}} s\left[m-m^{\prime}, l-l^{\prime}\right] .
$$

With $s[m, l]=0$ for $m<0$, this relation can be used to recursively calculate the TFMA parameters $b_{m, l}$ as

$$
b_{m, l}=\frac{1}{N} \sum_{m^{\prime}=0}^{m-1} \sum_{l^{\prime}=-L}^{L} \frac{m-m^{\prime}}{m} b_{m^{\prime}, l^{\prime}} s\left[m-m^{\prime}, l-l^{\prime}\right], \quad m=1, \ldots, M .
$$

This recursion is initialized by $b_{0, l}$, which is obtained as follows. We can write $H[n, m]=b_{0}[n] H^{\prime}[n, m]$, where $H^{\prime}[n, m]$ is monic $\left(H^{\prime}[n, 0] \equiv 1\right)$. Hence, the CTFC $s[m, l]$ of $H[n, m]$ equals the sum of the CTFCs of $b_{0}[n]$ and $H^{\prime}[n, m]$. Since $H^{\prime}[n, m]$ is monic, its CTFC vanishes for $m=0$, and thus the CTFC of $b_{0}[n]$ equals $s[0, l]$. Inverting the $z$-transform in the implicit CTFC definition (8) on the unit circle for $m=0$, we then obtain after some calculations

$$
\begin{aligned}
b_{0, l} & =\frac{1}{N} \int_{0}^{1} \exp \left(\frac{1}{N} \sum_{l^{\prime}=-\infty}^{\infty} s\left[0, l^{\prime}\right] e^{j 2 \pi l^{\prime} \xi}\right) e^{-j 2 \pi l \xi} d \xi \\
& \approx \frac{1}{N} \underset{n \rightarrow l}{\mathbb{F}} \exp \left(\underset{l^{\prime} \rightarrow n}{\mathbb{F}^{-1}} \tilde{s}\left[0, l^{\prime}\right]\right) .
\end{aligned}
$$

\subsection{Relation to the Evolutionary Cepstrum}

In [2], the evolutionary cepstrum (EC) of a possibly noncausal, non minimum phase LTV system was introduced. The EC is based on Zadeh's time-varying transfer function [11]

$$
L_{n}(z) \triangleq \sum_{m=-N / 2}^{N / 2-1} H[n, m] z^{-m}
$$

We note that $L_{n}(z)=L\left(e^{j \frac{2 \pi}{N} n}, z\right)$ (cf. (7) and (5)). Assuming that for all $n, L_{n}(z) \neq 0$ for $z \in \mathscr{R}$ where $\mathscr{R}=\{z|1-\varepsilon \leq| z \mid \leq 1+\varepsilon\}$ with a positive constant $\varepsilon$, the $\operatorname{EC} h[n, m]$ is defined implicitly as

$$
\log \left(L_{n}(z)\right)=\sum_{m=-\infty}^{\infty} h[n, m] z^{-m} .
$$

If the system is time-invariant, then $H[n, m]=H[m]$ and thus the $\mathrm{EC}$ reduces to the classical complex cepstrum.

An aliased version of the EC can be computed as

$$
\tilde{h}[n, m]=\underset{k \rightarrow m}{\mathbb{F}}-1 \log \left(\underset{m^{\prime} \rightarrow k}{\mathbb{F}} H\left[n, m^{\prime}\right]\right) .
$$

With (5), we can rewrite (13) in terms of the SF $S[m, l]$ :

$$
\tilde{h}[n, m]=\underset{k \rightarrow m}{\mathbb{F}}-1 \log \left(\underset{m^{\prime} \rightarrow k}{\mathbb{F}} \underset{l^{\prime} \rightarrow n}{\mathbb{F}^{-1}} S\left[m^{\prime}, l^{\prime}\right]\right) .
$$

Comparing with (10), we see that the (aliased) EC is the inverse DFT of the (aliased) CTFC:

$$
\tilde{h}[n, m]=\underset{l \rightarrow n}{\mathbb{F}^{-1}} \tilde{s}[m, l] .
$$

Let us now reconsider the general time-varying MA model (1), which is assumed such that the zeros of $L_{n}(z)$ are within the unit circle for all $n$. Based on the EC, the following recursion for the time-varying MA parameters $b_{m}[n]$ has been derived in [2]:

$$
b_{m}[n]=\sum_{m^{\prime}=0}^{m-1} \frac{m-m^{\prime}}{m} b_{m^{\prime}}[n] h\left[n, m-m^{\prime}\right], \quad m=1, \ldots, M,
$$


initialized by

$$
b_{0}[n]=e^{h[n, 0]}
$$

(however, in [2] a different initialization was used). With (14), one can show that within the aliasing approximation, our CTFC-based recursion (11), (12) can be derived from the EC-based recursion (15), (16) if the TFMA basis expansion (3) is imposed on the timevarying MA parameters $b_{m}[n]$. However, there are major differences regarding the number of parameters $b_{m}[n]$ and $b_{m, l}$ and the computational complexity of the two recursions, as discussed next.

\subsection{Computational Complexity}

The TFMA model $(2)$ uses $(2 L+1)(M+1)$ parameters $b_{m, l}$. Calculation of the CTFC requires $4 N$ FFTs of length $N$ and $N^{2}$ complex logarithms. The recursion (11) uses $\mathscr{O}\left(L^{2} M^{2}\right)$ multiplications. Thus, TFMA parameter calculation requires $\mathscr{O}\left(N \log N+L^{2} M^{2} / N\right)$ multiplications plus $N$ complex logarithms per signal sample.

The general time-varying MA model (1) uses $N(M+1)$ parameters $b_{m}[n]$. For EC calculation, $2 N$ FFTs of length $N$ and $N^{2}$ complex logarithms need to be computed. The recursion (15) requires $\mathscr{O}\left(N M^{2}\right)$ multiplications. Hence, the overall complexity per signal sample is $\mathscr{O}\left(N \log N+M^{2}\right)$ plus $N$ complex logarithms.

It is thus seen that for typical situations where $L^{2} \ll N$, TFMA parameter computation based on the CTFC recursion is far less numerically intensive than parameter computation for the general time-varying MA model based on the EC recursion. The reason is the smaller number of model parameters to be computed

\section{PARAMETER ESTIMATION}

We now discuss estimation of the TFMA parameters $b_{m, l}$ from one or several observed realizations of the nonstationary process $x[n]$.

\subsection{The CTFC of a Nonstationary Process}

The expected ambiguity function (EAF) of a nonstationary process $x[n]$ with correlation $R_{x}[n, m]=\mathbb{E}\left\{x[n] x^{*}[n-m]\right\}$ is defined as [5]

$$
\bar{A}_{x}[m, l] \triangleq \sum_{n=0}^{N-1} R_{x}[n, m] e^{-j \frac{2 \pi}{N} l n}=\underset{n \rightarrow l}{\mathbb{F}} R_{x}[n, m] .
$$

This is the SF of an LTV system with impulse response $R_{x}[n, m]$ (cf. (5)). The correlation of a TFMA process is given by

$$
R_{x}[n, m]=\sum_{m^{\prime}=0}^{M} \sum_{l=-L}^{L} \sum_{l^{\prime}=-L}^{L} b_{m^{\prime}, l} b_{m^{\prime}-m, l^{\prime}}^{*} e^{j \frac{2 \pi}{N}\left[\left(l-l^{\prime}\right) n+l^{\prime} m\right]},
$$

and thus

$$
\bar{A}_{x}[m, l]=N \sum_{m^{\prime}=0}^{M} \sum_{l^{\prime}=-L}^{L} b_{m^{\prime}, l^{\prime}} b_{m^{\prime}-m, l^{\prime}-l}^{*} e^{j \frac{2 \pi}{N}\left(l^{\prime}-l\right) m} .
$$

It follows that $\overline{A_{x}}[m, l]=0$ for $(m, l) \notin[-M, M] \times[-2 L, 2 L]$. We next consider the two-sided 2-D $z$-transform of $A_{x}[m, l]$ (cf. (7)),

$$
P_{x}\left(z_{1}, z_{2}\right) \triangleq \frac{1}{N} \sum_{m=-N / 2}^{N / 2-1} \sum_{l=-N / 2}^{N / 2-1} \bar{A}_{x}[m, l] z_{1}^{l} z_{2}^{-m} .
$$

Evaluation of $P_{x}\left(z_{1}, z_{2}\right)$ on the unit bicircle yields (cf. (9))

$$
P_{x}[n, k] \triangleq P_{x}\left(e^{j \frac{2 \pi}{N} n}, e^{j \frac{2 \pi}{N} k}\right)=\underset{m \rightarrow k}{\mathbb{F}} \underset{l \rightarrow n}{\mathbb{F}}{ }^{-1} \overline{A_{x}}[m, l],
$$

which can be interpreted as a time-varying power spectrum of $x[n]$ [5]. We then define the CTFC of the process $x[n]$, denoted by $a_{x}[m, l]$, implicitly as (cf. (8))

$$
\log \left(P_{x}\left(z_{1}, z_{2}\right)\right)=\frac{1}{N} \sum_{m=-\infty}^{\infty} \sum_{l=-\infty}^{\infty} a_{x}[m, l] z_{1}^{l} z_{2}^{-m} .
$$

An aliased version of the CTFC can be calculated as (cf. (10))

$$
\tilde{a}_{x}[m, l]=\underset{n \rightarrow l}{\mathbb{F}} \underset{k \rightarrow m}{\mathbb{F}}-1 \log \left(\underset{m^{\prime} \rightarrow k}{\mathbb{F}} \underset{l^{\prime} \rightarrow n}{\mathbb{F}}-1 \bar{A}_{x}\left[m^{\prime}, l^{\prime}\right]\right) .
$$

We now assume that the product of maximum delay $M$ and maximum Doppler $L$ satisfies $M L \ll N$, i.e., both the innovations system and the process $x[n]$ are strongly underspread [5]. It then follows that $e^{j \frac{2 \pi}{N}\left(l^{\prime}-l\right) m} \approx 1$ within the summation range of (17) since $\left|1-e^{j \frac{2 \pi}{N}\left(l^{\prime}-l\right) m}\right|=2\left|\sin \left(\frac{\pi}{N}\left(l^{\prime}-l\right) m\right)\right| \leq 4 \pi \frac{M L}{N}$. Hence, (17) can be approximated by a simple 2-D convolution of $b_{m, l}$ with $b_{-m,-l}^{*}$ :

$$
\overline{A_{x}}[m, l] \approx N \sum_{m^{\prime}=0}^{M} \sum_{l^{\prime}=-L}^{L} b_{m^{\prime}, l^{\prime}} b_{m^{\prime}-m, l^{\prime}-l}^{*} .
$$

With (6), (7), and (18), this yields $P_{x}\left(z_{1}, z_{2}\right) \approx L\left(z_{1}, z_{2}\right) L^{*}\left(\frac{1}{z_{1}^{*}}, \frac{1}{z_{2}^{*}}\right)$ and further $\log \left(P_{x}\left(z_{1}, z_{2}\right)\right) \approx \log \left(L\left(z_{1}, z_{2}\right)\right)+\left[\log \left(L\left(\frac{1}{z_{1}^{*}}, \frac{1}{z_{2}^{*}}\right)\right)\right]^{*}$. Thus,

$$
a_{x}[m, l] \approx s[m, l]+s^{*}[-m,-l],
$$

where, as before, $s[m, l]$ is the CTFC of the innovations system.

For a minimum phase TFMA model, $s[m, l]=0$ for $m<0$, and thus (22) implies

$$
a_{x}[m, l] \approx \begin{cases}s[m, l], & m>0 \\ 2 s[0, l], & m=0 \\ s^{*}[-m,-l], & m<0\end{cases}
$$

(note that $s^{*}[0,-l]=s[0, l]$ ). Thus, the TF cepstral recursion (11) can approximately be written in terms of $a_{x}[m, l]$ as

$b_{m, l} \approx \frac{1}{N} \sum_{m^{\prime}=0}^{m-1} \sum_{l^{\prime}=-L}^{L} \frac{m-m^{\prime}}{m} b_{m^{\prime}, l^{\prime}} a_{x}\left[m-m^{\prime}, l-l^{\prime}\right], \quad m=1, \ldots, M$,

and the expression (12) for the initialization parameter $b_{0, l}$ becomes

$$
b_{0, l} \approx \frac{1}{N} \underset{n \rightarrow l}{\mathbb{F}} \exp \left(\frac{1}{2} \underset{l^{\prime} \rightarrow n}{\mathbb{F}^{-1}} \tilde{a}_{x}\left[0, l^{\prime}\right]\right) .
$$

With (20), the relations (23) and (24) show how to calculate the TFMA parameters $b_{m, l}$ from the second-order statistics of $x[n]$ (EAF $\bar{A}_{x}[m, l]$ or, equivalently, correlation $\left.R_{x}[n, m]\right)$. They allow to estimate the $b_{m, l}$ from one or several realizations of $x[n]$ if $\bar{A}_{x}[m, l]$ is replaced by a suitable estimate. We note that a similar estimation procedure can be developed for EC-based parameter estimation.

\subsection{Estimation of the EAF}

Let us assume that $I$ realizations $x_{i}[n]$ have been observed. We propose the EAF estimator (cf. [3, 12])

where

$$
\hat{A}_{x}[m, l]=\Psi[m, l] \sum_{i=1}^{I} A_{x_{i}}[m, l],
$$

$$
A_{x_{i}}[m, l] \triangleq \sum_{n=0}^{N-1} x_{i}[n] x_{i}^{*}[n-m] e^{-j \frac{2 \pi}{N} l n}=\underset{n \rightarrow l}{\mathbb{F}}\left\{x_{i}[n] x_{i}^{*}[n-m]\right\}
$$

is the ambiguity function [12] of $x_{i}[n]$ and $\Psi[m, l]$ is a 2-D weight function that is designed such that it effectively covers the support region of $\bar{A}_{x}[m, l]$ and the corresponding nonparametric spectrum estimate $\hat{P}_{x}[n, k]=\underset{m \rightarrow k}{\mathbb{F}} \underset{l \rightarrow n}{\mathbb{F}^{-1}} \hat{A}_{x}[m, l]$ (cf. (19)) is nonnegative. Such a weight function can be constructed as [13]

$$
\Psi[m, l]=\sum_{j=1}^{K} \gamma_{j} A_{g_{j}}^{*}[m, l],
$$

where the $\gamma_{j}$ are the $K$ largest positive eigenvalues of the LTV system whose SF equals the indicator function of the EAF's support 

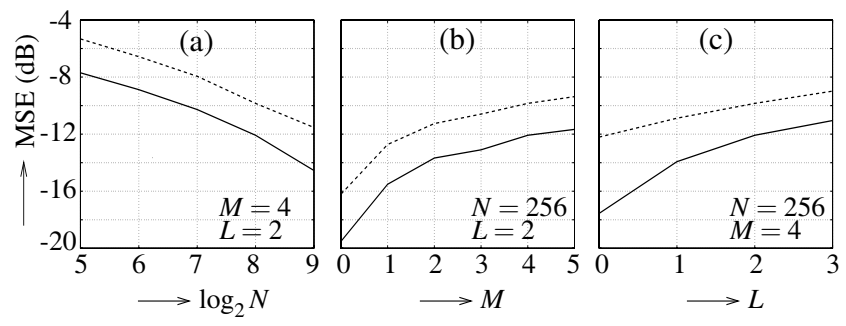

Figure 2: Normalized MSE (in dB) of our CTFC-based parameter estimator (solid line) and of the EC-based parameter estimator [2] (dashed line) for a TFMA process, plotted versus (a) signal length $N$, (b) delay model order $M$, and (c) Doppler model order $L$.

region and the $g_{j}[n]$ are the associated eigenfunctions. This presupposes knowledge of the EAF's support region (i.e., of $M$ and $L$ ).

\section{SIMULATION RESULTS}

We next assess the performance of our TFMA parameter estimator by means of numerical simulations.

TFMA Processes. We first consider TFMA processes with given parameters $b_{m, l}$, delay model order $M \in\{0,1, \ldots, 5\}$, Doppler model order $L \in\{0,1,2,3\}$, and signal length $N \in\{32,64,128,256$, $512\}$ (note that the process is more underspread for larger $N$ and/or smaller delay-Doppler order product $M L$ ). We applied our TFMA parameter estimator described in Section 4 by estimating the EAF from a single realization $(I=1)$ according to (25), (26) and evaluating the cepstral recursion (23), (24) based on the true model order $(M, L)$. The normalized MSE of the resulting parameter estimates (obtained by averaging over 50 such experiments) is shown as a function of $N, M$, and $L$ in Fig. 2. For comparison, we also show the MSE obtained with the EC-based parameter estimator [2] using the improved initialization (16). Note that in contrast to our estimator, the EC-based estimator does not require or make use of knowledge of the Doppler model order $L$.

Our estimator is seen to outperform the EC-based estimator for all $N, M$, and $L$, with gains between 2 and $5 \mathrm{~dB}$. The performance of both estimators improves with increasing $N$ and decreasing $M$ and $L$. Thus, as expected, parameter estimation is more accurate for a model with slower time variations or smaller maximum delay.

Non-TFMA Process. We next consider estimation of the timevarying spectrum $P_{x}[n, k]$ (see (19)) of a nonstationary process that does not conform to the TFMA model. The true spectrum $P_{x}[n, k]$ is depicted in Fig. 3(a). Fig. 3(b) shows a TFMA model based parametric spectrum estimate ${ }^{2} \hat{P}_{x}[n, k]=\left|N \underset{m \rightarrow k \rightarrow n}{\mathbb{F}} \underset{l \rightarrow n}{\mathbb{F}}-1 \hat{b}_{m, l}\right|^{2}$. Here, $\hat{b}_{m, l}$ denotes the TFMA parameter estimates obtained from a single realization $x[n]$ via our CTFC-based estimator using (optimum) model orders $(M, L)=(2,2)$. Finally, Fig. 3(c) shows a parametric spectrum estimate $\hat{P}_{x}[n, k]=\left|\underset{m \rightarrow k}{\mathbb{F}} \hat{b}_{m}[n]\right|^{2}$, where $\hat{b}_{n}[m]$ denotes the time-varying MA parameter estimates obtained from a single realization $x[n]$ via the EC-based estimator using (optimum) model order $M=1$. The normalized MSE of the spectrum estimates (obtained by averaging over 50 experiments) was $9.6 \%$ for the CTFCbased estimator and $19.0 \%$ for the EC-based estimator; note that this MSE also includes a systematic modeling error.

\section{CONCLUSIONS}

The time-frequency-moving-average (TFMA) model for nonstationary processes introduced in this paper has the advantage of being both physically intuitive (since it is formulated in terms of Doppler

\footnotetext{
${ }^{2}$ This spectrum estimate is based on the approximate expression

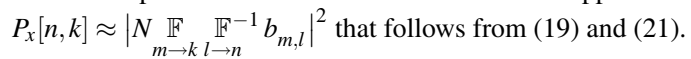
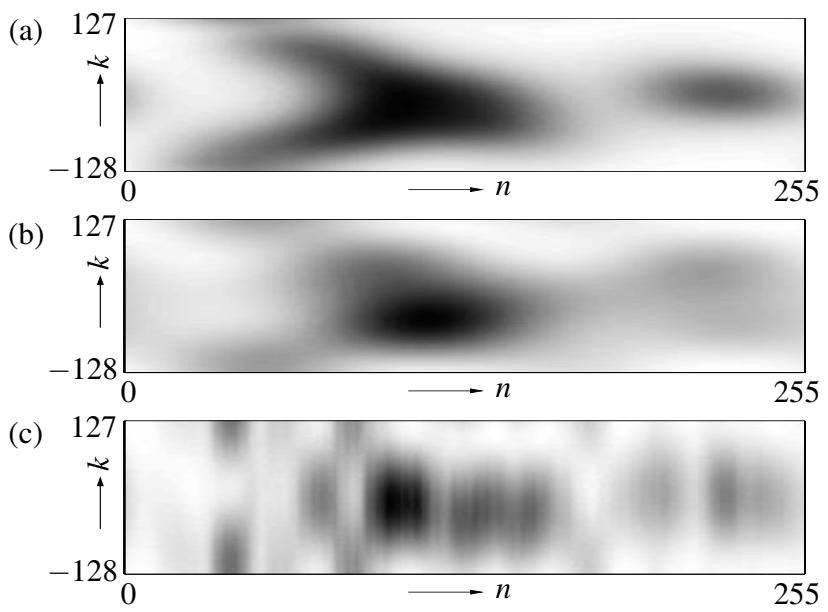

Figure 3: Parametric time-varying spectrum estimation: (a) True spectrum, (b) CTFC-based spectrum estimate, and (c) EC-based spectrum estimate.

shifts) and highly parsimonious. Based on a novel complex TF cepstrum, we derived a TF cepstral recursion that allows reliable and computationally efficient estimation of the TFMA parameters. Simulation examples illustrated the excellent performance of our model and estimator and their advantage over the evolutionary cepstrum approach proposed in [2]. The development of an automated procedure for TFMA model order selection and the extension of our parameter estimation technique to noisy observations are interesting topics for future research.

\section{REFERENCES}

[1] Y. Grenier, "Time-dependent ARMA modeling of nonstationary signals," IEEE Trans. Acoust., Speech, Signal Processing, vol. 31, pp. 899-911, Aug. 1983.

[2] A. Kaderli and A. S. Kayhan, "Spectral estimation of nonstationary ARMA processes using the evolutionary cepstrum," IEEE Signal Processing Letters, vol. 9, pp. 130-132, April 2002.

[3] M. Jachan, G. Matz, and F. Hlawatsch, "Time-frequency-autoregressive random processes: Modeling and fast parameter estimation," in Proc. IEEE ICASSP-2003, vol. VI, (Hong Kong), pp. 125-128, April 2003.

[4] P. Stoica and R. Moses, Introduction to Spectral Analysis. Englewood Cliffs (NJ): Prentice Hall, 1997.

[5] G. Matz and F. Hlawatsch, "Time-varying power spectra of nonstationary random processes," in Time-Frequency Signal Analysis and Processing: A Comprehensive Reference (B. Boashash, ed.), ch. 9.4, pp. 400-409, Oxford (UK): Elsevier, 2003.

[6] P. A. Bello, "Characterization of randomly time-variant linear channels," IEEE Trans. Comm. Syst., vol. 11, pp. 360-393, 1963.

[7] A. V. Oppenheim and R. W. Schafer, Digital Signal Processing. Englewood Cliffs, NJ: Prentice Hall, 1975.

[8] C. I. Byrnes, P. Enqvist, and A. Lindquist, "Cepstral coeffi cients, covariance lags, and pole-zero models for fi nite data strings," IEEE Trans. Signal Processing, vol. 49, pp. 677-693, April 2001.

[9] D. E. Dudgeon, "The existence of cepstra for two-dimensional rational polynomials," IEEE Trans. Acoust., Speech, Signal Processing, vol. 23, pp. 242-243, April 1975.

[10] R. Rom, "On the cepstrum of two-dimensional functions," IEEE Trans. Inf. Theory, vol. 21, pp. 214-217, March 1975.

[11] L. A. Zadeh, "Frequency analysis of variable networks," Proc. of IRE, vol. 76, pp. 291-299, March 1950.

[12] P. Flandrin, Time-Frequency/Time-Scale Analysis. San Diego (CA): Academic Press, 1999.

[13] F. Hlawatsch, G. Matz, H. Kirchauer, and W. Kozek, "Time-frequency formulation, design, and implementation of time-varying optimal fi lters for signal estimation," IEEE Trans. Signal Processing, vol. 48, pp. 1417-1432, May 2000. 